\title{
BMJ Open Leverage points to improve smoking cessation treatment in a large tertiary care hospital: a systems-based mixed methods study
}

\author{
Alex T Ramsey, ${ }^{\oplus 1}$ Donna Prentice, ${ }^{2}$ Ellis Ballard ${ }^{3}$ Li-Shiun Chen, ${ }^{1}$ Laura J Bierut ${ }^{1}$
}

To cite: Ramsey AT, Prentice D, Ballard $\mathrm{E}$, et al. Leverage points to improve smoking cessation treatment in a large tertiary care hospital: a systems-based mixed methods study. BMJ Open 2019;9:e030066. doi:10.1136 bmjopen-2019-030066

- Prepublication history and additional material for this paper are available online. To view these files, please visit the journal online (http://dx.doi. org/10.1136/bmjopen-2019030066).

Received 25 February 2019

Revised 15 May 2019

Accepted 6 June 2019
Check for updates

(C) Author(s) (or their employer(s)) 2019. Re-use permitted under CC BY-NC. No commercial re-use. See rights and permissions. Published by BMJ.

${ }^{1}$ Department of Psychiatry, Washington University School of Medicine in Saint Louis, Saint Louis, Missouri, USA

${ }^{2}$ Department of Research for Patient Care Services, BarnesJewish Hospital, Saint Louis, Missouri, USA

${ }^{3}$ Brown School of Social Work and Public Health, Washington University in Saint Louis, Saint Louis, Missouri, USA

Correspondence to Dr Alex T Ramsey; aramsey@wustl.edu

\section{ABSTRACT}

Objectives To generate system insights on patient and provider levers and strategies that must be activated to improve hospital-based smoking cessation treatment. Design Mixed methods study including a series of indepth group model building sessions, which informed the design of an online survey completed by healthcare providers and a structured interview protocol administered at the bedside to patients who smoke.

Setting Large, tertiary care hospital in the Midwestern United States.

Participants Group model building: 28 healthcare providers and 22 previously-hospitalised patients; Online survey: 308 healthcare providers; Bedside interviews: 205 hospitalised patients.

Primary and secondary outcome measures Hypothesisgenerating, participatory qualitative methods informed the examination of the following quantitative outcomes: patient interest versus provider perception of patient interest in smoking cessation and treatment; patient-reported receipt versus provider-reported offering of inpatient smoking cessation interventions; and priority ratings of importance and feasibility of strategies to improve treatment.

Results System insights included patients frequently leaving the floor to smoke, which created major workflow disruption. Leverage points included interventions to reduce withdrawal symptoms, and action ideas included nurse-driven protocols for timely administration of nicotine replacement therapy. Quantitative data corroborated system insights; for instance, $80 \%$ of providers reported that patients frequently leave the floor to smoke, leading to safety risks, missed assessments and inefficient use of staff time. Patients reported significantly lower rates of receiving any smoking cessation interventions, compared with provider reports (mean difference $=17.4 \%-33.7 \%$, $\mathrm{p}<0.001$ ). Although $92 \%$ of providers cited patient interest as a key barrier, only $4 \%$ of patients indicated no interest in quitting or reducing smoking.

Conclusions Engaging hospital providers and patients in participatory approaches to develop an implementation strategy revealed discrepant perceptions of patient interest and frequency of hospital-based treatment for smoking. These findings spurred adoption of standardised pointof-care treatment for cigarette smoking, which remains highly prevalent yet undertreated among hospitalised patients.

\section{Strengths and limitations}

- Using a systems science lens, this study integrated multiple data sources to inform more systematic provision of smoking cessation treatment practices in hospital settings.

- This study featured a rigorous mixed methods approach whereby hypothesis-generating qualitative data informed the design of quantitative instruments and guided interpretation of resulting quantitative data.

- The use of participatory approaches to engage healthcare provider and patient stakeholders identified provider and patient levers to be activated in a robust implementation strategy.

- Despite having many similarities to other healthcare systems in the nation, study findings are based in one large tertiary care hospital system in the Midwestern United States.

\section{INTRODUCTION}

Cigarette smoking remains the leading cause of preventable death worldwide. ${ }^{1-4}$ National efforts directed at reducing tobacco use have contributed to a decline in the proportion of combustible cigarette smokers. ${ }^{5}{ }^{6}$ However, smoking prevalence among those entering hospital settings remains much higher than in the general population, as those with chronic conditions have higher prevalence of smoking. ${ }^{7}$ Hospital settings therefore present an opportune time to promote cessation. ${ }^{9} 10$

Despite the existence of effective, Food and Drug Administration-approved medications for smoking cessation-including nicotine replacement therapy, varenicline and bupropion ${ }^{11-13}$ - which can be combined with brief counselling for optimal success, ${ }^{14}$ these treatments remain underutilised in inpatient settings. ${ }^{15-17}$ In our hospital, only $18 \%-24 \%$ of patients who smoke received smoking cessation pharmacotherapy during hospitalisation from 2010 to $2016 .{ }^{8}$ Further, 
these medications were often prescribed inconsistently between admitting services and subdemographic groups in our hospital. For instance, African Americans were $35 \%$ less likely than European Americans to receive smoking cessation pharmacotherapies during their hospital stay, which highlights concerning inequalities in hospital prescribing practices. ${ }^{8}$ Similar patterns were found in a study of smoking cessation pharmacotherapy rates among smokers hospitalised for an acute cardiac condition across 282 US hospitals; the median treatment rate was $22.3 \%$ and even lower among minority racial/ ethnic groups. ${ }^{17}$ Treatment rates across these hospitals were also highly variable, suggesting that hospitals may be using, with varying levels of success, different strategies to implement inpatient smoking cessation treatment programmes.

These treatment gaps and disparities indicate suboptimal care, as inpatient smoking cessation pharmacotherapy in combination with postdischarge treatment has been shown in meta-analyses to improve quit rates and is now considered the standard of care by Joint Commission. ${ }^{10}{ }^{15}$ Nevertheless, these treatment gaps and disparities signal that the implementation of smoking cessation treatment approaches remains a formidable challenge in hospital settings. Currently, it is not clear which patient and provider levers and strategies must be activated to improve smoking cessation treatment in hospital settings. Additionally, there is inadequate guidance on the best system-level implementation strategies to use to improve treatment delivery for hospitalised patients who smoke. The healthcare system requires pragmatic evidence to increase the likelihood that other hospitals use strategies that were robustly supported by rigorous yet relevant data and decrease the likelihood that hospitals use strategies without robust support. However, obtaining pragmatic, rigorous and relevant data may require novel uses of methods including more participatory, stakeholder-engaged approaches that integrate diverse types of data to gain a better understanding of the system-level gaps in care, high-leverage target areas to focus change efforts and specific strategies that can improve the treatment of patients who smoke. This study uses a systems science lens that integrates multiple data sources to inform more systematic provision of smoking cessation treatment practices in hospital settings, thereby using a novel approach to address a thorny problem that has challenged healthcare systems for decades.

\section{Study purpose}

The goal of the study was to understand determinants of the treatment gap in hospitalised patients who smoke and inform the development of stakeholder-supported strategies for improving smoking cessation treatment delivery in the inpatient setting. Building on our robust, validated electronic health record data demonstrating suboptimal smoking cessation pharmacotherapy prescription practices, ${ }^{8}$ this study integrated multiple data sources to inform more systematic provision of smoking cessation treatment practices in hospital settings. To our knowledge, this is the only study to engage with hospital patients and providers in a participatory process to identify the underlying system structure producing the treatment gap, collateral effects of this gap (eg, impacts on provider workflow), optimal leverage points and actionable strategies to yield consistent delivery of smoking cessation care in hospital settings. ${ }^{18-21}$

\section{METHODS}

This mixed methods study integrated qualitative and quantitative data sources including in-depth group model building sessions with healthcare providers and patients, ${ }^{21} 22$ followed by an online survey with healthcare providers and bedside interviews with patients.

\section{Phase I: group model building with healthcare providers and patients (qualitative work) \\ Participants and procedures}

Participants were recruited from Barnes-Jewish Hospital $(\mathrm{BJH})$, a large tertiary care hospital. We engaged two participant populations ${ }^{1}$ : $\mathrm{BJH}$ employees including physicians, nurses and support staff with patient contact and ${ }^{2}$ patients who had recently received care at BJH and who self-identified as current smokers at the time of their most recent hospital admission. To recruit healthcare providers, we requested programme directors and nursing supervisors of a diverse set of service linesincluding general surgery, internal medicine, neurology, oncology, orthopaedics, otolaryngology and psychiatry-to distribute recruitment materials to employees who may be eligible to participate. We excluded participants from the intensive care unit, emergency room and operating room due to lower relevance of the topic in these acute care settings. We also prioritised our active recruitment efforts within service lines likely to find the topic most relevant for their service delivery; therefore, some hospital services (eg, plastic surgery, urology) fell outside the scope of our recruitment efforts. To recruit patients who had been previously hospitalised at $\mathrm{BJH}$, we distributed a recruitment email through a research participant registry to potentially eligible individuals who smoke, posted a Facebook advertisement on the registry fan page listing, and hung printed flyers in the hospital. Those interested in participating were screened using a standardised telephone script.

We engaged 50 stakeholders in five group model building sessions comprised of patients (two sessions, $\mathrm{n}=22$ ); nurses, social workers and case managers (one session, $\mathrm{n}=14)$; nurse practitioners, hospitalists and pharmacists (one session, $\mathrm{n}=6$ ); and resident physicians (one session, $n=8)$. Using a standard process for synthesising the models, ${ }^{22-24}$ we then invited all participants in the initial sessions to reconvene for a model review that functioned to present a preliminary synthesis model for critique and refinement of the stakeholder-generated model (one session, $\mathrm{n}=16$ ). Patients and providers stemmed from a 
wide variety of hospital service lines, including cardiology, general surgery, internal medicine, neurology, obstetrics, oncology, orthopaedics, otolaryngology and psychiatry. We received participation from each service line from which we recruited. Sessions were conducted between late October 2017 and early February 2018.

Planning, conduct and analysis of the series of sessions were led by a core modelling team, including the principal investigator, $\mathrm{BJH}$ nursing partner and two experts in group model building and community-based system dynamics. Each group model building session utilised a facilitation team, which included the conveners/closers (principal investigator and nursing partner), primary modeller (lead expert in group model building), facilitators (approximately two support experts in group model building) and note takers (approximately two additional team members). The group model building team used scripts ${ }^{23}$ or a pre-defined set of exercises and behaviours to provide a semistructured environment for ${ }^{1}$ patient stakeholder groups to model a typical sequence of clinical encounters that may or may not result in patients being offered smoking cessation treatment and ${ }^{2}$ healthcare provider stakeholder groups to model a typical sequence of clinical decision points with regard to ordering and administering smoking cessation treatment and offering counselling in the hospital. Patient participants were then asked to identify factors that determine the likelihood of being offered and receiving smoking cessation treatment. Similarly, healthcare provider participants were asked to identify factors that determine the likelihood of offering smoking cessation pharmacotherapy and counselling, the likelihood of patients accepting pharmacotherapy and counselling, sources of decision-making and workflow barriers. All participants were then asked to prioritise the identified factors and position them as intervening variables within the modelled sequence of events.

\section{Mixed methods analytic approach}

We used an exploratory sequential design in which qualitative data from the group model building sessions informed the design of the quantitative online survey and patient bedside interviews. ${ }^{25}$ The qualitative data were hypothesis generating to further guide the development of the quantitative measures and interpretation of the resulting quantitative data.

\section{Phase II: online survey with healthcare providers (quantitative and qualitative)}

Participants and procedures

We recruited healthcare providers to complete an anonymous 10-min online survey by emailing a cover letter and survey link to administrative contact persons, programme directors and nursing staff who then distributed the email through their networks. Participants of the online survey included 308 providers (112 physicians, 196 nurses) from $\mathrm{BJH}$ with direct inpatient contact. Participants were located across a wide range of service lines, with internal medicine $(46 \%)$ and general surgery (22\%) being the most highly represented. As in phase I, we excluded participants from the intensive care unit, emergency room and operating room due to lower relevance of the topic in these acute care settings. Of physicians, $79 \%$ were resident physicians, and $21 \%$ were hospitalists. Of nurses, $47 \%$ were staff nurses, $30 \%$ nurse practitioners and $23 \%$ other types of nurses. Nurses and physicians were asked via quantitative assessments about their current smoking cessation treatment practices-namely the use of the '5As': Ask about tobacco use, Advise to quit smoking, Assess readiness for quit attempt, Assist with medication and counselling options and Arrange follow-up contact or referral, ${ }^{26-28}$ barriers to using these practices, perceived patient interest in various smoking cessation resources during an inpatient stay, perceived importance and feasibility of various potential strategies to improve practices and the frequency of workflow and safety issues related to hospitalised patient smoking. Qualitative data were obtained through an open-ended prompt to share 'final thoughts or comments on the topic of smoking cessation treatment at $\mathrm{BJH}$ '. The online survey remained open from late February 2018 to late March 2018.

\section{Phase III: bedside interviews with patients (quantitative) Participants and procedures}

We recruited hospitalised patients to complete a structured 5-min interview at the bedside. Whereas patients in phase I were recruited subsequent to their hospitalisation as necessary for convening the group model building sessions, patients recruited in phase III for the brief individualised interviews were still hospitalised yet nearing discharge. This facilitated accurate recall of events while minimising the risk of missing patients who had recently been discharged or delaying patients' ability to exit the hospital once discharged. We obtained reports daily during the month of May 2018 from the hospital electronic health record to identify potentially eligible patients and conducted all interviews during this time period. Participants of the bedside interviews included 205 inpatients who were categorised by electronic health record as current smokers and nearing hospital discharge. Participants were more often male (56\%) and Caucasian (59\%), with a median age of 54 years $(\mathrm{M}=50.25 ; \mathrm{SD}=15.22)$. These demographics appeared to be very similar to those of the larger population of $\mathrm{BJH}$ patients across the hospital who were current smokers during this time frame (May 2018). On average, participants smoked 14.0 cigarettes per day and had stayed in the hospital for 4.5 days within a variety of admitting services, including internal medicine (38\%), surgery (23\%), oncology (13\%), cardiology (12\%), neurology $(7 \%)$ and orthopaedics $(5 \%)$. Based on an existing questionnaire to compare patient and provider reports in a different context, ${ }^{26}$ patients were asked about their smoking behaviours (ie, verification of current smoker status prior to hospital admission, cigarettes per day and frequency of leaving the floor to smoke); ever and current e-cigarette use; smoking cessation care they had received during their current inpatient stay (ie, 
receipt of the 5As: ask, advise, assess, assist and arrange); interest in quitting smoking now, quitting smoking later, smoking less, and methods to quit smoking (eg, medications); number of past-year quit attempts; and the importance of various potential strategies to improve practices (eg, offering medication to every patient who smokes).

\section{Statistical analysis approach}

Data from the healthcare provider online surveys and patient bedside interviews were first analysed descriptively via frequencies and means. Patient-reported receipt of 5 As smoking cessation practices was examined in relation to provider reports using summary independent-sample t-test analyses. Multiple linear analyses were conducted to determine associations between medication receipt and the likelihood and frequency of leaving the floor to smoke, controlling for cigarettes per day and length of stay. Missing data across variables were minimal $(<2 \%$ for healthcare providers and $<3 \%$ for patients) and handled via pairwise deletion.

\section{Patient and public involvement}

During participant recruitment, patients were able to identify other potentially eligible individuals who smoke to be screened for enrollment. Through the participatory group model building sessions, patients generated key system insights that informed the research questions and outcome measures to be assessed in the subsequent online survey for healthcare providers and bedside interview protocol for patients. For instance, patients expressed significant frustration regarding being asked repeatedly about their smoking behaviours without being offered any help to quit smoking during their hospital stay. As a result, the research team prioritised questions in the quantitative instruments to assess the frequency of patient-and provider-reported delivery of smoking cessation interventions, as well as patient and provider reports of patient interest in receiving smoking cessation treatment while hospitalised. This research reflects a key step in developing a system-level intervention; therefore, patient input is contributory to ongoing research and practice improvements. We plan to disseminate results of this study to patients and other participants by presenting findings at local symposia and conferences that are well attended by patients, patient advocates, healthcare providers and the broader community. We will also present these findings during healthcare provider training workshops, whereby patients benefit through improved quality of smoking cessation care in the hospital.

\section{RESULTS}

\section{Phase I: group model building}

System insights-'what factors determine the likelihood that patient smoking will be treated?'

Based on factors prioritised by participants during group model building, the research team generated a multilevel (ie, individual, hospital, community and policy) understanding, referred to as system insights, that characterised the observed undertreatment of patients who smoke. Online supplementary file 1 illustrates the intentionally oversimplified backbone structure to which participants were responding and building on with content and context. These insights included provider reports of patients frequently leaving the floor to smoke, which created major workflow problems and enhanced provider receptivity to solutions framed to address 'nicotine withdrawal' rather than 'cessation'. A commonly reported scenario involved untreated hospital patients going outside to smoke, leading to missed assessments or procedures, which then prompted nurses to prioritise smoking cessation medications to prevent further workflow disruptions. Providers also reported a lack of awareness of resources and enthusiasm balanced with concerns about time, while patients reported infrequent receipt of smoking cessation support and preferences for non-judgemental communication (see table 1).

\section{Potential leverage points-'what targets could lead to major system-level improvements?'}

With these system insights providing the appropriate frame, the modelling group then identified potential leverage points to target for action. Leverage points refer to places within a complex system in which a small change can produce large changes in the overall system behaviour. ${ }^{29}$ Potential leverage points to address provider-reported insights included interventions framed as solutions to reduce nicotine withdrawal and subsequent workflow problems, education and decision support and a standardised approach to smoking cessation treatment. Potential leverage points to address patient-reported insights included transparency of patient interest and use of treatment and patient-provider rapport through more supportive cessation messaging (see table 1).

\section{Action ideas-'which specific strategies appear both highly important and feasible?'}

Finally, stakeholders used these potential leverage points as the frame for nominating potential concrete solutions and then prioritising them on perceived importance and feasibility in the hospital setting. Providers generated action ideas to address their own provider-reported insights and leverage points, including implementing nurse-driven protocols for timely administration of inpatient nicotine replacement therapy, using an assortment of provider training approaches, designing electronic health records to support point-of-care decisions and offering point-of-care advice, medication and links to outpatient counselling. Patients generated action ideas to address their own patient-reported insights and leverage points, including creating provider feedback systems, developing cessation plans for discharge, offering help to every patient who smokes and revamping hospital signage for more positive messaging (see table 1). These system insights, potential leverage points and action 


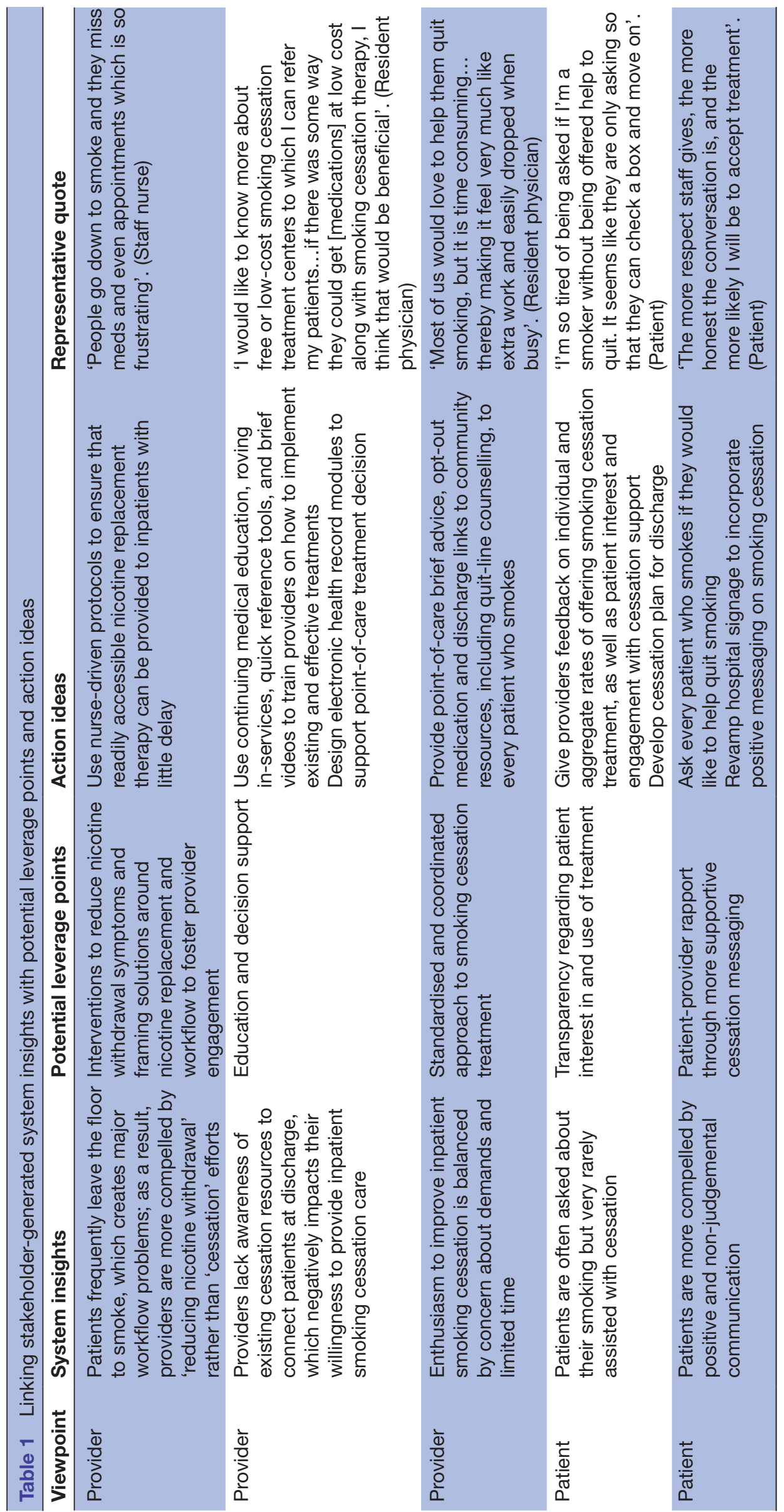


ideas were then examined more closely in the subsequent online survey with providers and bedside interviews with patients.

\section{Phase II: healthcare provider online survey}

\section{Qualitative data}

Open-ended responses from the provider sample were useful in expanding further on the group model building data. Key representative quotes are included in table 1 to reinforce themes from the group model building sessions.

\section{Quantitative data}

Rates of smoking cessation practice varied substantially across the 5As, with the majority of providers indicating regular completion of Ask (88\%), Advise (79\%), Assess (62\%) and Assist (63\%), with lower rates for Arrange (24\%). Additionally, $64 \%$ of providers reported that they often-to-always encourage the use of medication (eg, nicotine replacement therapy and varenicline) with patients for smoking cessation.

The most commonly cited barriers to providing treatment were patient lack of interest (92\%) and compliance $(92 \%)$, followed by lack of awareness of existing community resources for patient referral $(72 \%)$ and lack of time $(71 \%)$.

Approximately $80 \%$ of providers reported that patients often or very often leave the floor to smoke, and this frequently leads to patients posing a safety risk (75\% reported often or very often), assessments being missed (51\% reported often or very often) and staff time used to escort patients off the floor ( $42 \%$ reported often or very often).

The strategies rated as most important for improving smoking cessation treatment at the hospital were also the ones rated as most feasible. These top strategies included asking every patient who smokes if they want help $(86 \%$ endorsed as very or extremely important; $77 \%$ endorsed as very or extremely feasible), offering brief advice to every patient who smokes $(65 \%$ endorsed as very or extremely important; $56 \%$ endorsed as very or extremely feasible) and offering medication to every patient who smokes $(62 \%$ endorsed as very or extremely important; $52 \%$ endorsed as very or extremely feasible).

\section{Table 2 High patient demand to quit smoking}

\begin{tabular}{ll}
\hline Variables & $\begin{array}{l}\text { Patients, } \\
\text { n/N (\%) }\end{array}$ \\
\hline Interested in quitting now & $146 / 200(73)$ \\
\hline Interested in quitting now or later & $188 / 202(93)$ \\
Interested in quitting now or later or smoking & $193 / 202(96)$ \\
less & $118 / 201(59)$ \\
Past year quit attempt & $131 / 202(65)$ \\
Ever used e-cigarettes & $25 / 202(12)$ \\
\hline Currently used e-cigarettes &
\end{tabular}

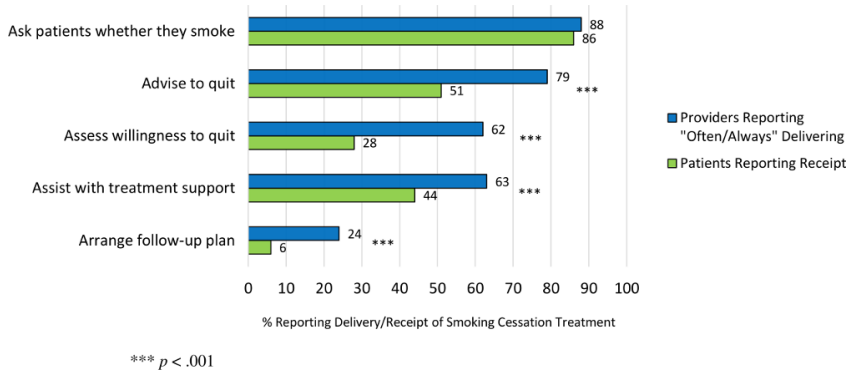

Figure 1 Providers and patients report discrepant rates of inpatient smoking cessation treatment.

\section{Phase III: patient bedside interviews}

Table 2 highlights high levels of patient smoking cessation interest and attempts. In particular, nearly three-fourths $(73 \%)$ were interested in quitting now, and nearly all $(96 \%)$ were interested in quitting or smoking less. Most patients $(59 \%)$ had tried quitting in the past year. Nearly two-thirds $(65 \%)$ had ever used e-cigarettes, and $12 \%$ currently used them.

Regarding comparisons between patient and provider reports of the 5As, patients reported rates similar to providers on Ask (mean difference $=-1.4 \% ; \mathrm{p}=0.653$ ); however, they reported much lower rates of receiving Advise (mean difference $=-27.5 \%$; $\mathrm{p}<0.001$ ), Assess (mean difference $=-33.7 \%$; $<<0.001$ ), Assist (mean difference $=-19.2 \% ; \mathrm{p}<0.001$ ) and Arrange (mean difference $=-17.4 \%$; $\mathrm{p}<0.001$ ) steps (see figure 1 ), as well as being provided with medication to quit smoking during their inpatient stay (mean difference $=-35.0 \%$; $\mathrm{P}<0.001$; see figure 2). Additionally, in contrast to $92 \%$ of providers citing patient interest as a key barrier to smoking cessation treatment, only $4 \%$ of patients indicated no interest in quitting at some point or smoking less, and only $27 \%$ of patients indicated no interest in quitting now.

Nearly one-third (31\%) of patients reported that they had left the floor to go smoke during their hospital stay, and nearly two-thirds of those who left did so multiple times per day. Overall, receipt of inpatient smoking cessation medication was not significantly associated with leaving the floor to smoke $(\mathrm{p}=0.331)$. However, among patients who left, those who did not receive smoking cessation medications were more likely to report leaving multiple times per day than those who received medications, controlling for cigarettes per day and length of stay $(\mathrm{OR}=3.3, \mathrm{p}=0.036)$.

Patients were well-aligned with providers regarding perceived importance of potential strategies to improve smoking cessation treatment in the hospital (see figure 3). The most highly rated strategies were to ask every patient who smokes if they want help $(76 \%$ endorsed as very or extremely important), offer medication to every patient who smokes $(67 \%)$ and offer brief advice to every patient who smokes $(58 \%)$. 


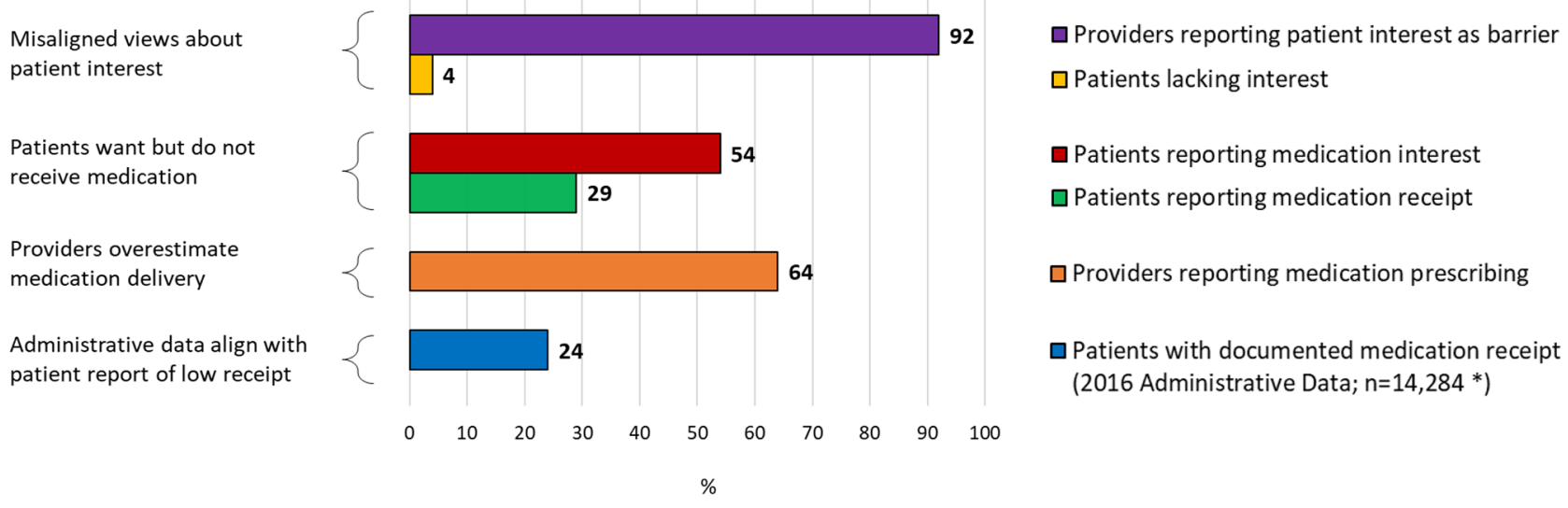

* Adapted from Srivastava et al., 2018 [8]

Figure 2 Despite high interest, fewer patients receive pharmacologic cessation treatment than indicated by providers.

\section{DISCUSSION}

Extensive research has examined the hospital as a prime setting to engage patients in smoking cessation treatment, as well as effective treatment approaches to employ in hospital settings, ${ }^{9-14}$ yet persistent treatment gaps signal formidable implementation challenges. ${ }^{15-17}$ This study uniquely employed a systems science lens to frame the implementation challenges and opportunities using a rigorous mixed methods approach to generate system insights, potential leverage points and specific strategies to improve the treatment of hospitalised patients who smoke. Key contributions of this research include (1) detailing an underutilised participatory, stakeholder-engaged process to yield hypothesis-generating qualitative data that informed the design and interpretation of quantitative data and (2) a robust set of provider and patient

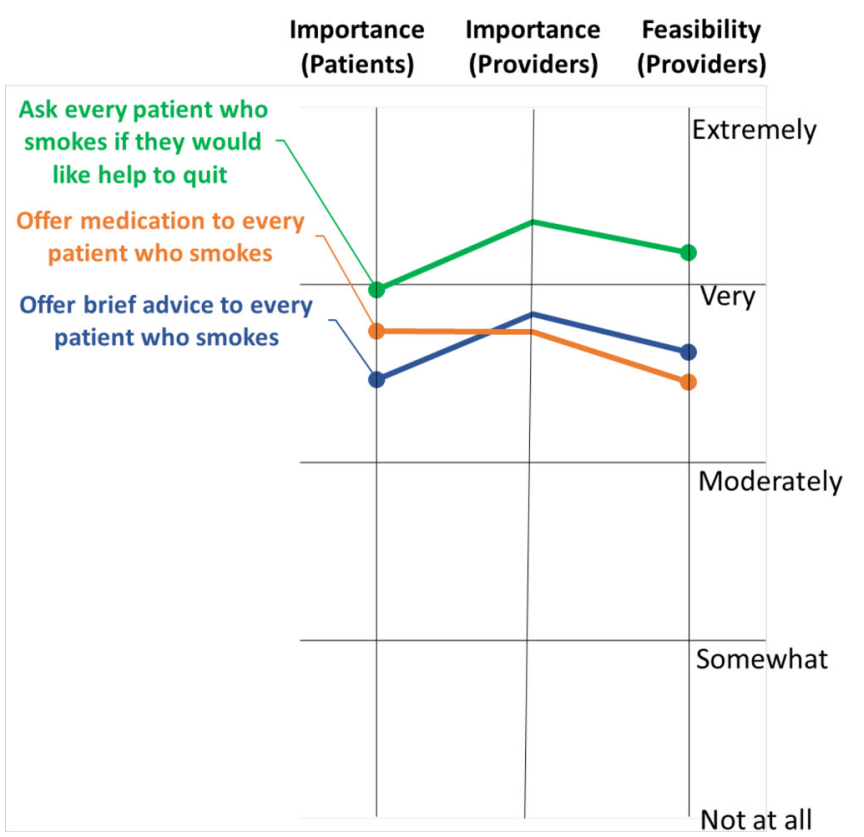

Figure 3 Potential strategies prioritised by importance and feasibility among stakeholders. levers to be activated in a multicomponent implementation strategy in future research.

Healthcare providers reported that patient smoking during a hospitalisation created significant workflow issues for hospital staff. Despite low current rates of smoking cessation pharmacotherapy prescribing, we found high patient demand to quit smoking. Provider-reported barriers centred on a perceived lack of patient interest, time, and awareness of existing resources; these barriers reflect aspects of motivation, opportunity, and capability, which have been identified as key determinants of behavioural change and fruitful targets for intervention. ${ }^{30} 31$

Despite the wealth of research on inpatient smoking and hospital-based cessation treatment, far fewer studies have focused on potential collateral effects of inpatient smoking and treatment gaps, such as impeded provider workflow characterised by missed assessments and procedures, misuse of staff time and potential safety concerns, as found in the current study. Importantly, providers were much more receptive to and compelled by approaches to prevent the chain of events involving nicotine withdrawal, patients leaving the floor to smoke, and workflow problems, as opposed to approaches framed as promoting smoking cessation among hospitalised patients. This finding has significant implications for approaches to engaging hospital providers in the treatment of inpatients who smoke.

Whereas previous research found that nearly one in five smokers admitted to a hospital smoked cigarettes during their hospital stay, ${ }^{32}$ the rate was nearly one in three among smokers sampled in our hospital setting. Interestingly, based on patient reports, receipt of nicotine replacement therapy was not associated with whether or not patients left the floor to smoke at least once; however, patients receiving nicotine replacement therapy were more likely to have only left the floor to smoke once. Therefore, it is possible that patients may have been receiving nicotine replacement therapy in response to leaving the floor to 
smoke (eg, missing patient prompts the provider to offer treatment for nicotine withdrawal), thereby reducing the likelihood that patients subsequently left due to nicotine withdrawal during their hospital stay. This hypothesis would require further testing, including an establishment of temporal precedence to demonstrate the risk of patient smoking before and after receiving nicotine replacement therapy in the hospital setting.

Regarding points of (mis)alignment between patient and provider perceptions, both groups reported high rates of asking patients whether or not they smoke. However, patients reported much lower rates of receiving any smoking cessation support and much higher levels of interest in cessation, compared with provider reports. This finding replicates recent research in mental health settings ${ }^{26}$ and was corroborated by qualitative data, which characterised the patient perception that providers frequently ask about smoking behaviours, yet no actions result from these inquiries. Improved alignment of perceptions could benefit patient-provider rapport and increase acceptance of smoking cessation treatments when offered-opportunities which were all raised by patients during group model building discussions.

Finally, patients and providers agreed that the most important strategies were to ask every patient who smokes if they want help and offer medication and brief advice to every patient who smokes. Providers also found these to be the most feasible potential strategies, despite the patient reports that these were not frequently occurring. While seen as feasible, providers may perceive the need for a hospital-wide programme that expects, supports and reinforces the practice of offering treatment to every patient who smokes. As noted in a recent systematic review, ${ }^{31}$ standardised implementation of this type of opt-out programme that leverages lighter-touch pointof-care support may in fact ease provider burden and workflow. In addition, the alignment between patients and providers in ratings of importance and feasibility give additional credence to the viability of implementing these proposed strategies in hospital settings.

Limitations of this study include being based in one large tertiary care hospital system in the Midwestern United States. However, there are indications that this hospital system is largely representative of other systems in the nation. For instance, researchers recently found smoking cessation medication rates of $22.3 \%$ across 282 US hospitals, ${ }^{17}$ nearly identical to our rate of approximately 22\% across years 2010-2016. In addition, patient recruitment for the group model building sessions was limited to a research participant registry; as a pool of patients who are willing to be contacted about research studies, participants from this registry may differ somewhat from patients at-large. For the online survey, the link was distributed by the primary contacts of hospital divisions, and it was not possible to determine how many healthcare providers received the opportunity to complete the survey. As a result, we were unable to ascertain response rate and therefore cannot rule out the possibility of sampling bias. Finally, patients and providers from psychiatric services may have different perceptions about smoking cessation treatment compared with other patient and provider groups. However, only two participants from psychiatry were included in the exploratory phase of our study (phase I), and no participants from psychiatry were included in phases II and III in which we compared provider and patient reports of treatment (ie, 5As) offering and receipt.

\section{CONCLUSION}

Our findings have led our hospital to adopt standardised, lighter-touch yet higher-reach approaches to smoking cessation treatment, supported by provider feedback and simplified decision support and enabled through the electronic health record system. ${ }^{9} 2033$ Prior reviews and studies frequently highlight the importance of directly targeting hospital systems, including integrating key performance indicators into electronic health records, to improve the delivery of hospital smoking cessation care and the sustainability of those improvements. ${ }^{35}$ Despite the local system changes, the primary contribution of these findings is in providing generalisable evidence to help other researchers, providers and hospital administrators to prioritise the use of implementation strategies that were robustly supported across each phase of our mixed methods study. The potential leverage points we identified point to the following specific strategies:

1. Create a standardised and coordinated approach to smoking cessation treatment: provide point-of-care brief advice, opt-out medication and discharge links to community resources, including quit-line counselling, to every patient who smokes.

2. Foster provider engagement by identifying and framing interventions as solutions to reduce nicotine withdrawal and subsequent workflow problems related to patients leaving the floor to smoke: use nurse-driven protocols to ensure that readily accessible nicotine replacement therapy can be provided to inpatients with little delay.

3. Offer positive, supportive and non-judgemental messaging to patients: revamp hospital signage to incorporate positive messaging on smoking cessation and focus on boosting confidence and motivation to quit in patients who smoke ${ }^{36}$.

4. Improve awareness, knowledge, self-efficacy and attitudes: use continuing medical education, roving in-services and quick reference tools to train providers on existing and effective treatments and how to implement them.

5. Increase transparency regarding patient interest in and use of treatment: give providers feedback on ongoing performance and aggregate rates of patient-reported readiness to quit and engagement with quit-line counselling to foster awareness and accountability.

Engaging hospital stakeholders through a process of self-identification of approaches to addressable problems presents opportunities to fit high-leverage challenges with sustainable, contextually appropriate solutions. Findings 
from this study inform engagement with healthcare provider and patient stakeholders in the development and implementation of proposed strategies to facilitate consistent delivery of smoking cessation treatment practices in the hospital setting.

Contributors AR, L-SC and LJB conceptualised and designed the study. AR and DP led participant recruitment and data collection. EB led the group model building sessions and synthesis of qualitative data. ATR analysed the qualitative and quantitative data and drafted the initial manuscript. AR, DP, EB, L-SC and LJB contributed to manuscript revisions and approved the final manuscript.

Funding Research reported in this paper was supported by National Institute on Drug Abuse (NIDA) grants K12DA041449, R01DA036583, R01DA038076, National Cancer Institute (NCI) grants U19CA203654, P30CA091842 and P30CA091842-16S2 and a grant from the Foundation for Barnes-Jewish Hospital.

Competing interests LJB is listed as an inventor on Issued U.S. Patent 8080371 , 'Markers for Addiction' covering the use of certain SNPs in determining the diagnosis, prognosis and treatment of addiction. The other authors have no financial disclosures.

Ethics approval This study was approved by an institutional review board in the Human Research Protection Office at Washington University in St. Louis (IRB ID: 201703162).

Provenance and peer review Not commissioned; externally peer reviewed.

Data sharing statement Additional data may be available by emailing aramsey@ wustl.edu.

Open access This is an open access article distributed in accordance with the Creative Commons Attribution Non Commercial (CC BY-NC 4.0) license, which permits others to distribute, remix, adapt, build upon this work non-commercially, and license their derivative works on different terms, provided the original work is properly cited, appropriate credit is given, any changes made indicated, and the use is non-commercial. See: http://creativecommons.org/licenses/by-nc/4.0/.

\section{REFERENCES}

1. Samet JM. Tobacco smoking: the leading cause of preventable disease worldwide. Thorac Surg Clin 2013;23:103-12.

2. Bauer UE, Briss PA, Goodman RA, et al. Prevention of chronic disease in the 21st century: elimination of the leading preventable causes of premature death and disability in the USA. Lancet 2014;384:45-52.

3. Yoon PW, Bastian B, Anderson RN, et al. Potentially preventable deaths from the five leading causes of death-United States, 20082010. MMWR Morb Mortal Wkly Rep 2014;63:369-74.

4. General $\mathrm{S}$. The health consequences of smoking -50 years of progress: A report of the Surgeon General. https://www. surgeongeneral.gov/library/reports/50-years-of-progress/index.html (Accessed 22 Jun 2018)

5. Centers for Disease Control and Prevention. Trends in current cigarette smoking among high school students and adults, United States, 1965-2014. http://www.cdc.gov/tobacco/data_statistics/ tables/trends/index.htm (Accessed 22 Jun 2018).

6. America's Health Rankings. Health measures in the United States 2018 annual report. https://www.americashealthrankings.org/explore/ annual/measure/Overall (Accessed 14 Dec 2018).

7. Froehlich-Grobe K, Jones D, Businelle MS, et al. Impact of disability and chronic conditions on health. Disabil Health J 2016;9:600-8.

8. Srivastava AB, Ramsey AT, McIntosh LD, et al. Tobacco use prevalence and smoking cessation pharmacotherapy prescription patterns among hospitalized patients by medical specialty. Nicotine Tob Res 2018.

9. Nahhas GJ, Wilson D, Talbot V, et al. Feasibility of implementing a hospital-based "opt-out" tobacco-cessation service. Nicotine Tob Res 2016;19:937-43.

10. Rigotti NA, Clair C, Munafò MR, et al. Interventions for smoking cessation in hospitalised patients. Cochrane Database Syst Rev 2012:5.
11. Cahill K, Stevens S, Perera R, et al. Pharmacological interventions for smoking cessation: an overview and network meta-analysis. Cochrane Database Syst Rev 2013:5.

12. Stead LF, Perera R, Bullen C, et al. Nicotine replacement therapy for smoking cessation. Cochrane Database Syst Rev 2012:11.

13. Keating GM, Lyseng-Williamson KA. Varenicline. Pharmacoeconomics 2010;28:231-54.

14. Stead L, Lancaster T. Combined pharmacotherapy and behavioural interventions for smoking cessation. J Evid Based Med 2012;5:242.

15. Fiore MC, Goplerud E, Schroeder SA. The Joint Commission's new tobacco-cessation measures--will hospitals do the right thing? $N$ Engl J Med 2012;366:1172-4.

16. Katz DA, Stewart K, Paez M, et al. "Let Me Get You a Nicotine Patch": Nurses' Perceptions of Implementing Smoking Cessation Guidelines for Hospitalized Veterans. Mil Med 2016;181:373-82.

17. Pack QR, Priya A, Lagu TC, et al. Smoking cessation pharmacotherapy among smokers hospitalized for coronary heart disease. JAMA Intern Med 2017;177:1525-7.

18. Diez Roux AV. Complex systems thinking and current impasses in health disparities research. Am J Public Health 2011;101:1627-34.

19. Powell BJ, Beidas RS, Lewis CC, et al. Methods to improve the selection and tailoring of implementation strategies. J Behav Health Serv Res 2017;44:177-94.

20. Geerligs L, Rankin NM, Shepherd HL, et al. Hospital-based interventions: a systematic review of staff-reported barriers and facilitators to implementation processes. Implement Sci 2018;13:36.

21. Zimmerman L, Lounsbury DW, Rosen CS, et al. Participatory system dynamics modeling: Increasing stakeholder engagement and precision to improve implementation planning in systems. Adm Policy Ment Health 2016;43:834-49.

22. Hovmand PS. Community Based System Dynamics. New York, NY: Springer, 2014.

23. Hovmand PS, Andersen DF, Rouwette E, et al. Group model-building 'scripts' as a collaborative planning tool. Syst Res Behav Sci 2012;29:179-93.

24. Luna-Reyes LF, Martinez-Moyano IJ, Pardo TA, et al. Anatomy of a group model-building intervention: building dynamic theory from case study research. Syst Dyn Rev 2006;22:291-320.

25. NIH Office of Behavioral and Social Sciences. Best practices for mixed methods research in the health sciences. 2nd ed. Bethesda: National Institutes of Health, 2018.

26. Chen LS, Baker T, Brownson RC, et al. Smoking cessation and electronic cigarettes in community mental health centers: patient and provider perspectives. Community Ment Health J 2017;53:695-702.

27. Fiore MC, Baker TB. Treating smokers in the health care setting. $N$ Engl J Med Overseas Ed 2011;365:1222-31.

28. Fiore MC, Jaén CR, Baker TB, et al. Treating Tobacco Use and Dependence: 2008 Update: US Department of Health and Human Services, 2008.

29. Meadows D. Leverage Points: Places to intervene in a system: Sustainability Institute, 1999.

30. Michie S, Atkins L, West R. The Behavior Change Wheel: A Guide to Designing Interventions. Great Britain: Silverback Publishing, 2014.

31. Sharpe T, Alsahlanee A, Ward KD, et al. Systematic review of clinician-reported barriers to provision of smoking cessation interventions in hospital inpatient settings. J Smok Cessat 2018;13:233-43.

32. Regan S, Viana JC, Reyen M, et al. Prevalence and predictors of smoking by inpatients during a hospital stay. Arch Intern Med 2012;172:1670-4.

33. Bernstein SL, Rosner J, DeWitt M, et al. Design and implementation of decision support for tobacco dependence treatment in an inpatient electronic medical record: a randomized trial. Trans/ Behav Med 2017;7:185-95.

34. Rice VH, Heath L, Livingstone-Banks J, et al. Nursing interventions for smoking cessation. Cochrane Libr 2017:12.

35. Slattery C, Freund M, Gillham K, et al. Increasing smoking cessation care across a network of hospitals: an implementation study. Implementation Science 2015;11:28.

36. Streck JM, Chang Y, Tindle HA, et al. Smoking cessation after hospital discharge: Factors associated with abstinence. $J$ Hosp Med 2018;13:774-8. 